\title{
NONINVASIVE HEMOGLOBIN LEVEL AND HEART RATE SENSOR SYSTEM FOR AUTOMATIC TREADMILL SPEED CONTROL
}

\author{
A.Kavithamani ${ }^{1} \&$ V. Manikandan ${ }^{2}$
}

Abstract- Persons having low hemoglobin concentration of blood should use the treadmill at proper speed to avoid risk of heart diseases. Hence it is important to find the level of hemoglobin of the person before exercising. A method to control the treadmill speed automatically with respect to the person's hemoglobin level and heart rate is presented in this paper. The hemoglobin concentration and the heart rate are measured noninvasively using Light Emitting Diode (LED) based sensor system to adjust the speed of the treadmill. The noninvasively measured hemoglobin level and heart rate of the person were verified with the laboratory test results. This system is mainly useful for the rehabilitation program of the anemic patients. Keywords - treadmill speed control, hemoglobin, heart rate, noninvasive method.

\section{INTRODUCTION}

Hemoglobin $(\mathrm{Hb})$ is the important component in human blood as it transports oxygen from the lungs to all parts of our body. The normal range of $\mathrm{Hb}$ ranges from 14 to 18 for men and 12 to 16 for women, with any reading below 10 causes serious concern. When the $\mathrm{Hb}$ concentration in the blood is less than a defined level, it is said to be anaemia which reduces oxygencarrying capacity of red blood cells. If anemia becomes severe it causes fatigue, weakness and dizziness.

Anemic people have a higher risk of having heart diseases as compared to those without anemia. The condition of people having heart disease will become worse if they have anemia also [1]. Hb is significantly associated with exercise performance. Patients with low $\mathrm{Hb}$ will have poor exercise capacity and would be at high risk of cardiovascular events [2].

Cardiovascular changes depend on the type of exercise and severity of exercises. Change in blood pressure and heart rate while walking in treadmill are more compared to bicycle ergometer exercise for a given equivalent oxygen uptake values due to more sympathetic activation [3]. Since over exercising of the body causes harmful effects on the heart rate, it is important to control the speed of the treadmill to reduce the stress on the person [4]. In this proposed system both Hb level and heart rate of the person are measured noninvasively and the speed of treadmill is varied accordingly.

This paper is organized as follows. Section II gives the block diagram of the proposed systems and its explanation. Section III explains the hardware model of the proposed system. Results are discussed in Section IV. Section V concludes the proposed system along with its novelty.

\section{BLOCK DIAGRAM OF PROPOSED SYSTEM}

The block diagram of the proposed noninvasive $\mathrm{Hb}$ level and heart rate sensor system for automatic treadmill speed control is shown in figure 1. It contains $\mathrm{Hb}$ level and heart rate sensor, Arduino Uno board (microcontroller), Permanent Magnet Direct Current (PMDC) motor of the treadmill, its power and driver circuit. The signal from $\mathrm{Hb}$ level and heart rate sensor is passed to the Arduino UNO microcontroller. If the $\mathrm{Hb}$ level of the person is in normal (greater than $10 \mathrm{~g} / \mathrm{dl}$ ), then the microcontroller will check the heart rate and based on that it changes the duty cycle of the pulse width modulated (PWM) signal which is sent to the motor driver circuit to control the speed of the motor. Otherwise, the microcontroller will reduce the duty cycle of the PWM signal irrespective of the heart rate to reduce the speed of the treadmill. Hence, the speed of the motor changes according to the $\mathrm{Hb}$ level and heart rate. The measured heart rate, $\mathrm{Hb}$ level and the duty cycle which is set by the microcontroller are displayed.

\footnotetext{
${ }^{1}$ Department of EEE, Coimbatore Institute of Technology, Coimbatore, Tamilnadu, India

${ }^{2}$ Department of EEE, Coimbatore Institute of Technology, Coimbatore, Tamilnadu, India
} 


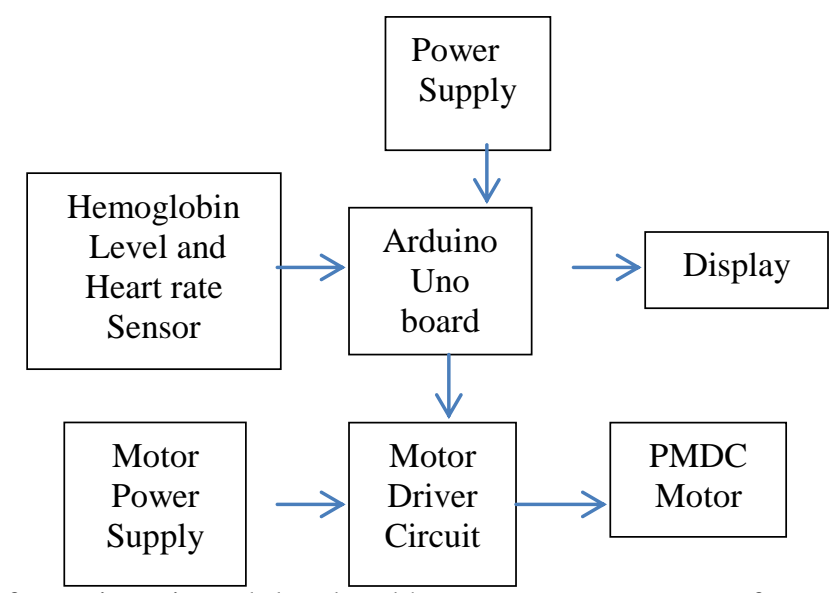

Figure 1. Block diagram of non-invasive $\mathrm{Hb}$ level and heart rate sensor system for automatic treadmill speed control

\subsection{Hemoglobin Level And Heart Rate Sensor}

The block diagram of $\mathrm{Hb}$ level and heart rate sensor shown in figure 2 consists of LED, photodetector and signal conditioning unit.

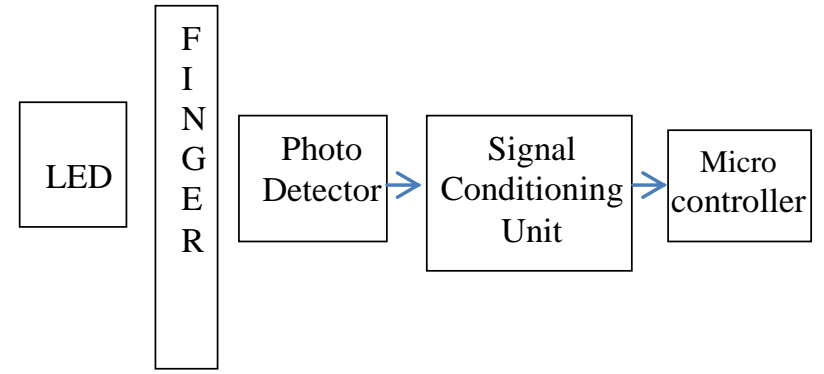

Figure 2. Block diagram of $\mathrm{Hb}$ level and heart rate sensor

\subsection{Hemoglobin measurement}

The proposed noninvasive measurement of $\mathrm{Hb}$ is based on Beer-Lambert law which states that absorbance is proportional to the concentration of the absorbing molecules, the length of the light path through the medium and the molar extinction coefficient.

$\mathrm{A}=\varepsilon \mathrm{cl}$

where A- Absorbance; $\varepsilon$ - molar extinction coefficient for the absorbing material;

$\mathrm{c}$ - concentration of the absorbing solution; 1 - light path in the absorbing material;

Hemoglobin consists of both oxy-hemoglobin and deoxy-hemoglobin. The absorption spectra of both oxy and deoxyhemoglobin are shown in figure 3 [6]. In the wavelength of 700nm, the extinction coefficient of deoxy-hemoglobin exceeds the extinction coefficient of the oxy-hemoglobin. Similarly, in the wavelength of the 900nm extinction coefficient of oxyhemoglobin exceeds the extinction coefficient of the deoxy-hemoglobin. But in the wavelength of 800nm, both the oxy and deoxy-hemoglobin have the same extinction coefficient. Hence LED light of wavelength $800 \mathrm{~nm}$ (Infrared) is used for this noninvasive measurement of $\mathrm{Hb}$ level. When the light is passed through the finger, both oxy and deoxy-hemoglobin absorb light proportional to the $\mathrm{Hb}$ level. The non-absorbed light is received by a photodetector.

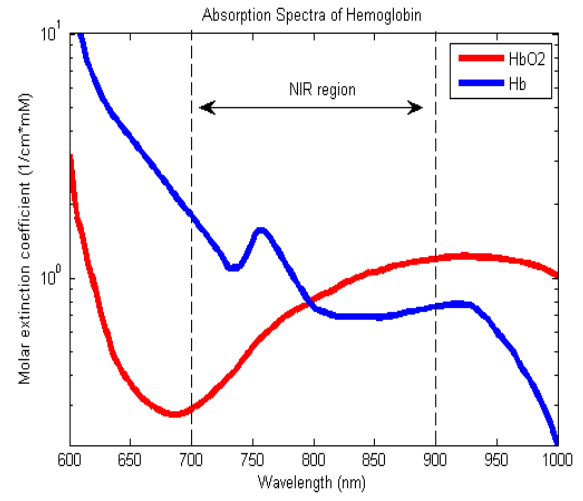

Figure 3. Absorption spectra of hemoglobin 
The photo detector fixed behind the finger converts the light signal into electrical signal. An amplifier which is added directly after the photo detector makes the signal detectable. The photo detector used in the proposed system is OPT101. The output voltage increases linearly with light intensity. The spectral responsivity of OPT101 is shown in figure 4 . It can be found that the photodetector OPT101 has higher spectral responsivity for 800nm wavelength. Hence this photodetector is suitable for the proposed system.

The maximum voltage and minimum voltage of the light signal from photodetector are measured and then the difference between maximum and minimum value is taken as amplitude. Arduino UNO microcontroller is programmed to determine the amplitude of the signal obtained from the photodetector. $\mathrm{Hb}$ level is inversely proportional to the amplitude obtained. An interrupt is sent to sample the output signal of the photodetector at every 0.04 seconds to calculate the maximum and minimum value, and the amplitude as illustrated in the flow chart shown in figure 5.

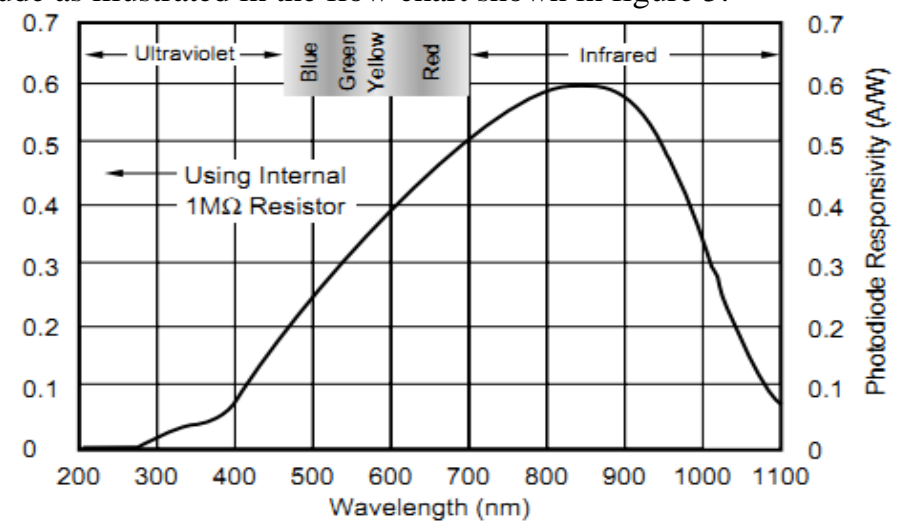

Figure 4. Spectral responsivity of OPT101

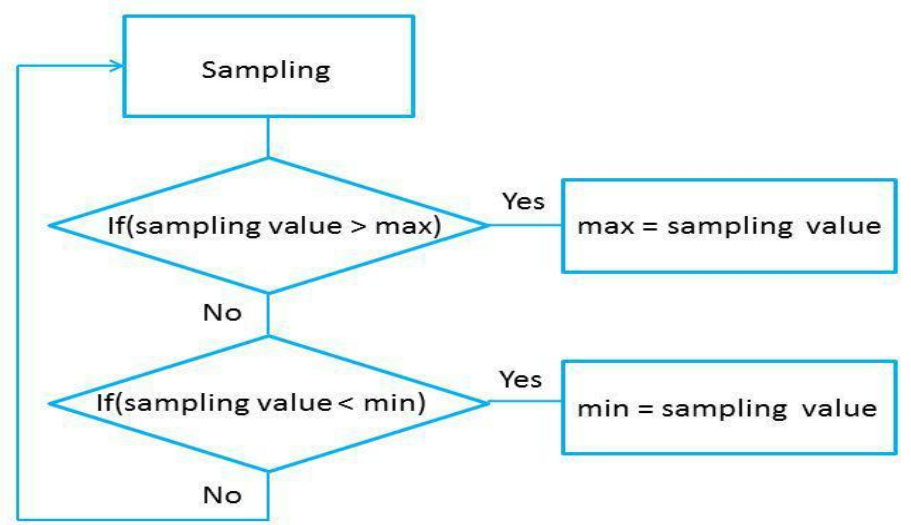

Figure 5. Flowchart for the measurement of maximum and minimum values

\subsection{Heart rate measurement}

Since the arteries contain more blood during the systolic phase of heart than during the diastolic phase due to an increased diameter, some oscillations (intensity changes) were produced in the output of the photodetector. These intensity changes are the so-called photoplethysmograph (PPG) waves (shown in figure 6). The heart rate is determined from these intensity changes. Heart rate measurement is done by analyzing the frequency of AC component of voltage signal acquired by sensor system.

A two-stage signal conditioning circuit is used for removing the noise and DC component from the received signal. Filtering at each stage is accompanied by amplification of the filtered output signal. The time period between two peak detection is calculated using the microcontroller and the frequency is determined. Frequency is the number of cycles (beats) per second. As the heart rate is the number of beats per minute, the frequency is multiplied by 60 to get the heart rate.

The algorithm for determining the heart rate is shown in figure 7. The basic idea is that every period in the output signal of the photodetector crosses a certain value, called set point. The cross of the set point could be up-down or down-up. In this work, a up-down cross point is chosen. This is found by comparing current sampling value and last sampling value with the set point value. If the last sampling value is bigger than set point value and the current sampling value is smaller than set point value, the signal has a up-down cross of the set point. And then the sampling time between two up-down crosses is counted. As the signal frequency is equal to sampling frequency over the sampling times, the heart rate is obtained by multiplying the signal frequency by 60 . 


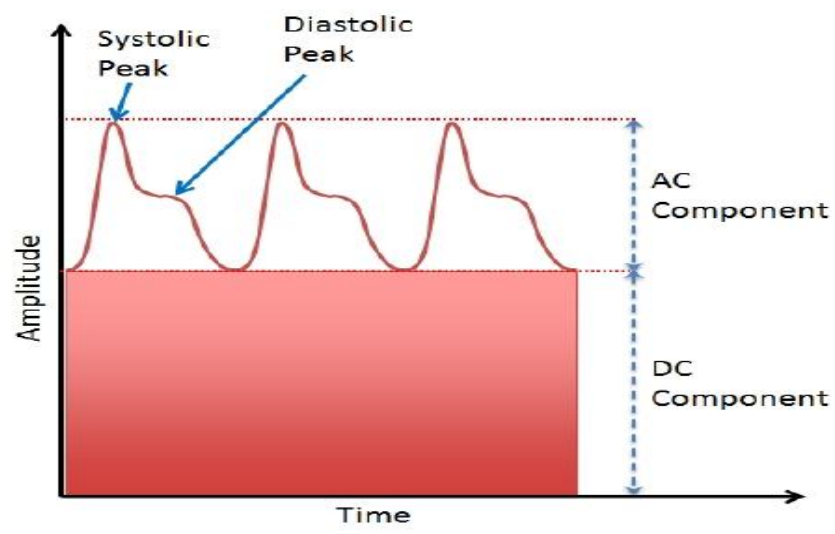

Figure 6. Output signal from photo detector

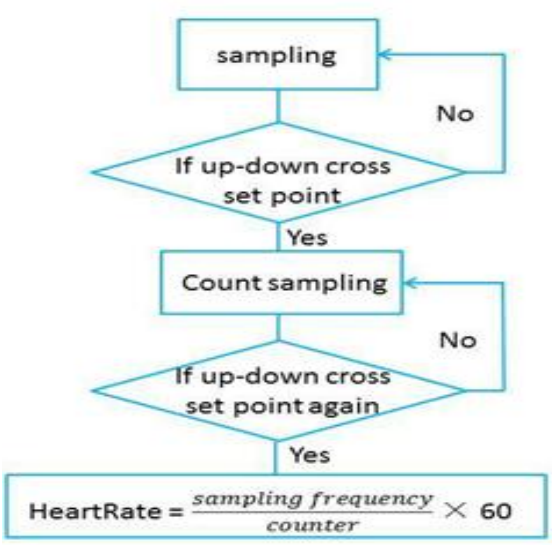

Figure 7. Flowchart for the measurement of heart rate

\subsection{PMDC And Its Control}

A permanent magnet DC (PMDC) motor is used in the proposed prototype model. It can produce very high torque at low speeds and hence it is used for treadmill application. Its speed-torque characteristics is more linear and predictable. Table 1 shows duty cycle of PWM signal to be generated by the microcontroller according to the Hb level and heart rate.

Table - 1 Duty Cyle of PWM Signal

\begin{tabular}{|c|c|c|c|}
\hline S. No. & Hb Level $(\mathrm{g} / \mathrm{dl})$ & Heart Rate (bpm) & Duty Cycle of the PWM Signal (\%) \\
\hline 1 & \multirow{4}{*}{$\begin{array}{l}\text { Normal } \\
\text { (Above 10) }\end{array}$} & $<60$ & 90 \\
\hline 2 & & 60 to 75 & 75 \\
\hline 3 & & 75 to 100 & 50 \\
\hline 4 & & $>100$ & 25 \\
\hline 5 & Below Normal (less than 10 ) & - & 25 \\
\hline
\end{tabular}

\section{HARDWARE MODEL}

The proposed system is first modeled and simulated using Proteus professional simulation software. After verifying the results the hardware setup is developed. It includes the $\mathrm{Hb}$ level and heart rate sensor system, signal conditioning unit (band pass filter), $12 \mathrm{~V}$ dual power supply, permanent magnet DC motor, microcontroller, and a CRO. The hardware of Hb level and heart rate sensor system shown in figure 8 consists of LED and a photo detector placed on either side of the finger clip. 


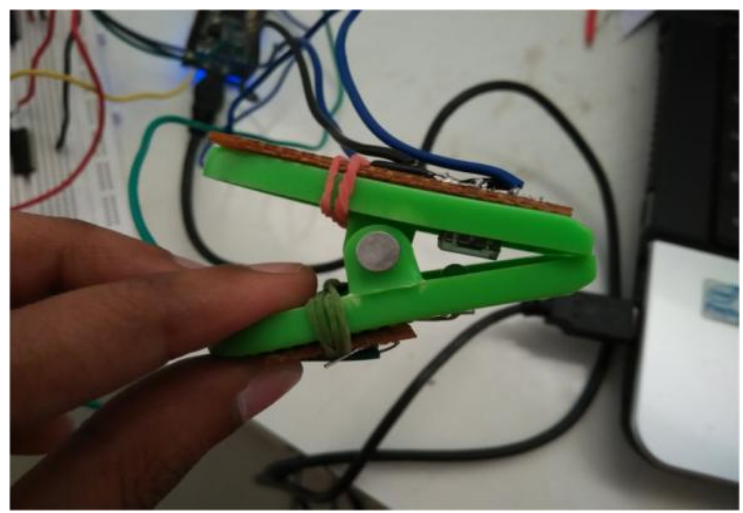

Figure 8. Hardware of $\mathrm{Hb}$ level and heart rate sensor system

Arduino microcontroller is programmed to generate a PWM signal with the value of duty cycle specified as in Table 1 according to the measure $\mathrm{Hb}$ level and heart rate. The proposed hardware model is shown in figure 9 along with the output waveform obtained from photodetector available on the CRO screen.

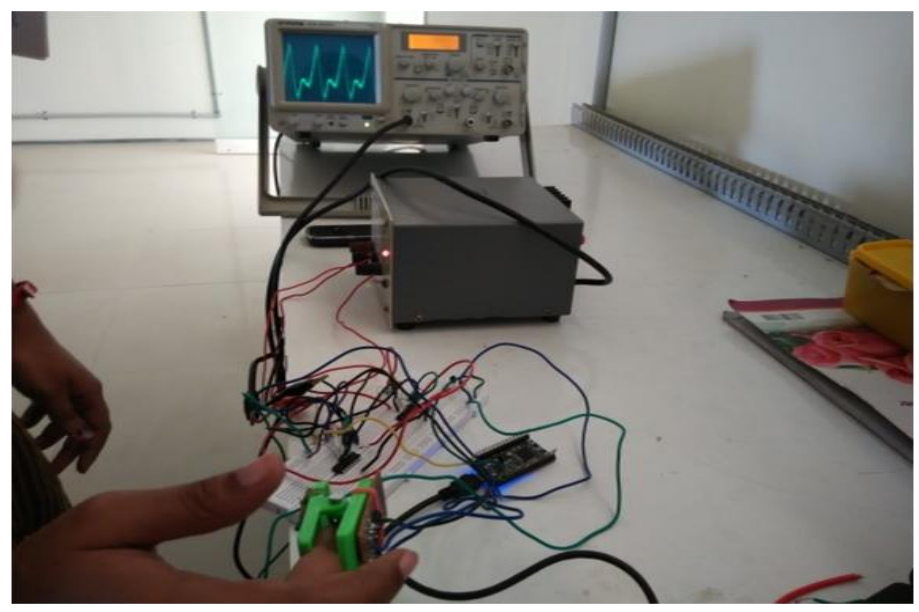

Figure 9. Hardware model of the proposed system

\section{RESULTS AND DISCUSSIONS}

Ten samples are considered to validate the proposed system. The actual $\mathrm{Hb}$ level and heart rate of these persons are obtained from the laboratory test results and they are compared with the results obtained from the proposed system. The comparison between actual reading and observed reading along with percentage error and the duty cycle of the PWM signal generated by the microcontroller according to Hb level and heart rate are shown in Table 2 in which DC refers to the duty cycle of the PWM signal.

Table - 2 Comparison between Actual and Observed Parameters of the Proposed System

\begin{tabular}{|c|c|c|c|c|c|c|c|}
\hline \multirow[t]{2}{*}{ S. No. } & \multicolumn{3}{|c|}{ Hemoglobin $(\mathrm{g} / \mathrm{dl})$} & \multicolumn{3}{|c|}{ Heart Rate (Bpm) } & \multirow[t]{2}{*}{$\mathrm{DC}(\%)$} \\
\hline & Actual & Observed & $\%$ Error & Actual & Observed & $\%$ Error & \\
\hline 1 & 7.1 & 7.08 & 0.28 & 69 & 66 & 4.34 & 25 \\
\hline 2 & 13.02 & 12.98 & 0.31 & 79 & 80 & -1.26 & 50 \\
\hline 3 & 12.6 & 12.65 & -0.4 & 70 & 68 & 2.86 & 75 \\
\hline 4 & 11.8 & 11.54 & 2.2 & 73 & 70 & 4.11 & 75 \\
\hline 5 & 15.5 & 15.62 & -0.77 & 72 & 73 & -1.39 & 75 \\
\hline 6 & 13.3 & 13.38 & -0.6 & 65 & 67 & $\begin{array}{l}-3.08 \\
\end{array}$ & 75 \\
\hline 7 & 11.5 & 11.72 & -1.91 & 72 & 74 & -2.78 & 75 \\
\hline 8 & 8.5 & 8.41 & 1.06 & 76 & 74 & 2.63 & 25 \\
\hline 9 & 9.3 & 9.4 & -1.07 & 75 & 78 & -4.00 & 25 \\
\hline 10 & 10.8 & 10.85 & -0.46 & 82 & 84 & -2.44 & 50 \\
\hline
\end{tabular}


From Table 2 it can be shown that the maximum percentage error between actual $\mathrm{Hb}$ level reading and observed $\mathrm{Hb}$ level reading is 2.2 and for heart rate is 4.34 . The proposed system is able to control the speed of the treadmill automatically according to the $\mathrm{Hb}$ level and heart rate of the person using the treadmill by generating PWM signals of various duty cycles.

\section{CONCLUSION}

Many methods are already available to control the treadmill speed automatically with respect to the person's heart rate. The main contribution of this work is, along with the heart rate, the $\mathrm{Hb}$ level is also considered to control the speed of the treadmill. In the proposed noninvasive method, ejection of blood is not required and there is no risk of infection. The cost of the sensor system is very less when compared with the chemical components required for invasive measurement of $\mathrm{Hb}$. Also in this proposed system a sensor which can be fixed in fingertip is designed which facilitates online monitoring. This system is mainly useful for the anemic patients to exercise on the treadmill without any risk.

\section{REFERENCES}

[1] Aryeh Shander, Lawerence T.Goodnough, Mazyar Javidroozi, Michael Auerbach, Jeffrey Carson, William B. Ershler, Mary Ghiglione, John Glaspy and Indu Lew, "Iron Deficiency Anemia - Bridging the Knowledge and Practice Gap", Journal of Transfusion Medicine Reviews, Elsevier, vol.28, Issue 3, July 2014, pp.156-166, doi:10.1016/j.tmrv.2014.05.001.

[2] Lipinski MJ, Dewey FE, Biondi-Zoccai GG, Abbate A, Vetrovec GW and Froelicher VF, "Hemoglobin levels predict exercise performance, STsegment depression, and outcome in patients referred for routine exercise treadmill testing”, Journal of Clinical Cardiology, vol. 32, Issue 12, pages E22 - E31, December 2009.

[3] Dr. Ravikiran Kisan MD, Dr. Swapnali Ravikiran Kisan MD, Dr. Anitha OR MD and Dr. Chandrakala SP MD, “Treadmill and Bicycle Ergometer Exercise: Cardiovascular Response Comparison", Global Journal of Medical Research, vol. 12, Issue 5, Version 1.0, June 2012, Online ISSN: 22494618 \& Print ISSN : 0975-5888.

[4] Greeshma.C.R and Dr. N. P. Ananthamoorthy, "Automatic Treadmill Speed Control with Heart Rate", International Journal for Trends in Engineering \& Technology”, vol. 3 Issue 1, pp. 149-153. - January 2015 - ISSN: 2349 - 9303.

[5] Jens Kraitl, Ulrich Timm, Hartmut Ewald and Elfed Lewis, "Non-invasive measurement of blood components", Proceedings of Fifth International Conference on Sensing Technology. pp.253-257, 2011.

[6] Rajashree Doshi and Anagha Panditrao, "Noninvasive optical sensor for Hemoglobin Determination", International Journal of Engineering Research and Applications (IJERA) vol. 3, Issue 2, pp.559-562, March -April 2013, v ISSN: 2248-9622.

[7] Toshiyo Tamura, Yuka Maeda, Masaki Sekine and Masaki Yoshida, "Wearable Photoplethysmographic Sensors_-Past and Present", Electronics 2014, vol.3, pp. 282-302,doi:10.3390/electronics3020282. 